\title{
Does chance really favor (only) the prepared mind? The role of MRI tissue-tagging in solving a most vexing problem for the interventionalist
}

\author{
Robert W. W. Biederman ${ }^{1}[$
}

Received: 20 July 2021 / Accepted: 22 July 2021 / Published online: 15 August 2021

(c) The Author(s), under exclusive licence to Springer Nature B.V. 2021

I remember it as if it was yesterday. I was sitting at the AHA meeting having lunch with Leon Axel, MD, PhD, while he was still at U Penn. I was finishing my Cardiovascular Fellowship and had long been fascinated by his then relative recent "discovery" of a new way to measure intramyocardial deformation as we called it then, now more succinctly described as strain. The story he told was having walked into the MRI lab and seeing some strange black line darting across the heart. As the heart moved, so did the dark line, in perfect synchrony with the underlying cardiac motion. In a Eureka moment, he realized that this was a new tool for tracking intra-myocardial motion. Serendipity had shown her face. Having been interested for decades in cardiac deformation, this emblematic moment confirmed Louis Pasteur's adage, 'Chance (does) favor the prepared mind'.

Interestingly, the surprised MRI technologist could neither recollect nor reproduce how she had performed such magic! So the story goes, Leon said by the end of the evening, he had deconstructed the sequence and defined the 'error'. By the next morning, he was able to perfectly recreate the 'artifact'. That was the start of feverish activity in his lab that culminated in the ability to lay down perfect prepulse-saturation bands, the actual source of the error, and space them as a series of parallel lines [1]. Within a short period of time, he and other investigators were able to non-invasively place simultaneous orthogonal grids,

This comment refers to the article available online at https://doi. org/10.1007/s10554-021-02355-4.

Robert W. W. Biederman

RBIEDERM@wpahs.org

https://

www.ahn.org/specialties/cardiovascular-institute/cardiac-mri

1 Cardiovascular Magnetic Resonance Imaging, Allegheny General Hospital, Temple University School of Medicine, Allegheny Health Network, Carnegie Mellon University, 320 E. North Avenue, Pittsburgh, PA 15212, USA radio-frequency tissue tagging (tags) that represented a grid pattern that deformed as the underlying cardiac muscle moved. From this initial work, we are now able to follow the intricate details of the fine intra-cardiac contractile motion [2]. It did not take long for other researchers to quickly grasp the value of this happenstance and further develop the technique. In a short time this led to unique insights into the intricacies of cardiac motion in health and disease along with a myriad of other applications.

To the unacquainted, reminiscent of Einstein's erasing Newton's theories, Leon Axel erased in an evening, generations of laboratory teams struggling to understand cardiac mechanics. This included myself, right after college, having spent 3 years in Blase Carabello 's lab in Charleston, South Carolina, personally carefully implanting into the beating open-heart animal model piezo-electric crystals one at a time to allow careful tracking of their complex motions [3, 4]. My job was eliminated in an instant. However, crystal work was important; it set the stage for MRI tissue tagging. Yet, as described to me by the late Nat Reichek, M.D. the very act of placing those crystals within the heart destroyed the very contractile machinery that one intended to understand. Typically, however it should be known, that one could measure generally no more than one or two endocardial and/ or epicardial segments per beating heart. Thus, Axel's one evenings' serendipitous observation initiated a huge leap forward in the understanding of non-invasive cardiac mechanics. Suddenly in one-fell- swoop literally hundreds or even thousands of grid tag-lines could be tracked, measuring not just $1 \mathrm{D}$ or $2 \mathrm{D}$ but $3 \mathrm{D}$ strain divided into endocardial, mesocardial and epicardial strain. Further, they could be resolved into circumstantial, radial and longitudinal strain and integrated to assess circumferential-longitudinal strain to more intuitively represent cardiac torsion/untwisting. In the late 1990's and 2000's, our lab and others were heavily invested in detailing nearly every myocardial motion that could be imagined. Meanwhile, Alistair Young, PhD., in Auckland 
(NZ), developed and popularized the unique principal strain representation making it possible to measure shear strain and a host of metrics via finite element analysis [2].

The knowledge gained informed our understanding of myocardial blood flow as it relates to direct and indirect myocardial contraction encompassing concepts such as cross fiber shortening and adjacent/remote myocardial contributions. This shed light on situations that were pivotal in our understanding of how endocardial infarction could occur yet be invisible to both conventional MRI and echocardiography-and surprisingly even to the interventional cardiologistwith quite normal chamber performance along with preserved locally and, of course, adjacent/ remote contraction. Many times, for instant, patients with markedly elevated CKs/MB's paired with dramatic STEMI's had far better than expected LVEF that was only properly understood using Cardiac MRI tissue tagging. For the record, I will state that Cardiac MRI tissue tagging pre-dated echocardiographic strain and even speckle tracking by several decades. But, imitation is indeed the sincerest form of flattery.

Even now our comprehension of the impact of infarct in both experimental and clinical medicine has been greatly advanced by our decades-long use of tagging that has as its origin in the prescient observation by Leon Axel. Likewise, our understanding of hypertensive heart disease [5, 6], HCM [7], AS/AR [8] and even pericardial interactions in constrictive pericarditis [9] have been highly informed by tagging as have the effects on regional function of a number of important pharmacologic agents used for treatment, such as ACE-inhibitors, beta blockers, dobutamine infusion and ARBs $[10,11]$. In the cardiomyopathies, myocardial tissue tagging has permitted more reliable assessment of the vast heterogeneity of segmental function. Finally, initial applications of myocardial tagging to assess right ventricular regional function in hypertrophied ventricles with and without complex congenital abnormalities have generated advances in understanding of effects of hypertrophy on left and right ventricular function.

Our interventional cardiology colleagues unknowingly owe a great deal of their abilities to grasp the complexities of preservation of cardiac function during, shortly following and chronically after AMI due to these intrepid tissue tagging studies.

So in this month's Journal, we learned that another group of investigators have seemingly stumbled on their own Eureka moment, employing intramyocardial radio-frequency tissue tagging to investigate local cardiac strains following angioplasty in high-risk cases [12].

Knaapen and colleagues from Amsterdam University (The Netherlands) embarked on an auspicious undertaking. For several decades, the concept of Chronic Total Occlusion (CTO) has been heavily debated. Whether, as the acronym suggests, a myocardial bed chronically subtended by a $100 \%$ occluded artery could not only be viable but could respond to the often superhuman, efforts to restore blood flow was the subject of intense debate. Indeed, a cottage industry has grown to supply the mechanical devices and approaches (ADR, AWE, RDT, RWE and various laser strategies) to assist the interventionalists in this task. Intriguing and often paradoxical but compelling studies supported each side of the argument $[13,14]$. Proponents and detractors alike of the 'Open Artery Hypothesis' could find science to support their beliefs. Indeed, at international meetings, the two camps were often devolved in contentious discussions and frank animus concerning the orthodoxy of recanalization.

Limitations of other investigators' approaches, including some of my colleagues, to conclusively prove or disprove the CTO theory, often hinged on features such as the lack of viability quantification, the inability to measure effective collateral blood physiology or correct assignment of artery subtending myocardium and other imperfections of this field [15]. Herein, the current authors carefully address these germane issues via their combined use of tagging and late gadolinium enhancement (LGE) to: (1) formally quantitate via full width-half maximum method the extent of viable and non-viable transmural muscle mass in order to assess the expected improvement in contraction, (2) demonstrate that collaterals, if present, are understood to be functional if there is no scar, and (3) assessment of right or left dominance a priori integrated within the contractile territory.

The critical step that these authors took to understand whether CTO was advisable was to integrate viability and tagging to discern whether the myocardium was viable and had the opportunity to improve if blood flow was effectively restored. Assessing efficacy of the procedure via tagging as the more pristine measure of cardiac contraction, rather than EF was the cardinal step the authors took to deduce the fate of the CTO hypothesis. For the reader it is critical that this concept be understood clearly. Classic measures to define post-PCI improvement have necessarily, but unfortunately, relied on LVEF; the for-better-or-worse workhorse tool of the cardiologist. The disadvantages of using LVEF is its' imprecision at the local level, along with its' well-known preload and afterload dependence, and the abject failure to demonstrate gross measures of improved cardiac function decried the CTO proponents for decades. Similarly, a more focused approach looking at regional mechanics via echocardiography was typically limited to measurements of either global longitudinal strain (GLS) or regional endocardium shortening (radial strain) with or without speckle-tracking. Rarely were both or all three performed simultaneously by echocardiography. Herein, the authors of the current manuscript included radial, circumferential and longitudinal strain in a rather Herculean effort in 150 patients with a CTO, before and 3 months following successful PCI. In bye-gone days, often a whole day of manual contouring, tracking and 
painstakingly assiduous effort for just one or two patients was required with loss of one's hairline-yet invariably, a gain of glasses. Fortunately, the work of many has made these contemporary analyses more automated and now a bit less arduous.

The authors reasoned if there was going to be a significant improvement in any of the local, adjacent or remote myocardial strain tensors, it would not be missed when employing a tissue tagging approach. It would be a binary phenomenon. If opening a chronically occluded artery was to ultimately be shown to be mechanically beneficial, tissue tagging would be the one tool to detect it. Critically, there would be a winner and a looser in this Olympic CTO-PCI match. And there was. The authors unequivocally demonstrated that despite restoration of blood flow to viable myocardium as defined by LGE, the viability 'Gold Standard' for greater than 2 decades, the winner of this marathon was to those who were the detractors of open artery hypothesis; CTO demonstrated no value. Were the authors satisfied? No. To assure that it was not a photo-finish the authors assessed LGE viability at multiple thresholds of $25,40,50,60$ and even $75 \%$ thresholds and still CTO-PCI lost. No matter how the judges reviewed the data, despite appeal, and even in that myocardium that was most likely to improve with near or full transmural viability, there was no clinically relevant improvement in LV function. Despite partitioning for LVEF above or below $50 \%$ in a desperate attempt to try to find a cohort where CTO-PCI might prevail demonstrated similar results; CTO-PCI suffered defeat.

To be fair, one might raise a question as to why in this cohort, $23 \%$ of the patients were asymptomatic? Consideration of risk: benefit ratios, given the known high complication rate of CTO attempts, would suggest that that cohort might experience significant risk with little chance of benefit. As it is, it seemed disadvantageous to consider CTOPCI at the onset [16].

But, we should ask: should restoration of partial or complete regional contraction be the yardstick in which to judge the ultimate success of a CTO-PCI approach? The definition of viability in this case is predicated on a return of contractile function. As the case may be, viability can be in the eye of the beholder. For instant, should restoration of an alternate cardiac physiologic variable be a reasonable surrogate? One of the often suggested benefits of the open artery hypothesis is that, even if myocardial substrate does not ultimately contract, there may be non-contractile benefits such as prevention of outward expansion, decreasing myocyte fibrosis and even more favorable preservation of electrical circuitry decreasing VT or SCD. Even reduction in softer endpoints such heart failure might be considered. Herein Dr. Knaepen and his colleagues are to be praised for their work in classical mechanical approaches to disprove once and for all the notion that CTO restores viability as contemporarily defined by restoration of cardiac mechanical contraction. Proponents of CTO may have to look elsewhere for continued support to buttress their beliefs; it is not to be found in this current work. The ability, as alluded to earlier, to detect contractile improvement, if it existed, is unparalleled. If not detectable by CMR strain; it does not exist. I believe that the failure to demonstrate with the highest resolved imaging tool known to interrogate myocardial mechanics, MRI radio-frequency tissue-tagging, a concept no doubt considered by Axel after his serendipitous discovery, will result in an abandonment of this CTO-PCI field, similar to how Magellan's circumnavigating the globe exactly 500 years ago this year delivered the death-blow to the felonious 'the earth is flat' belief.

\section{Declarations}

Conflict of interest The author declare that there is no conflict of interest.

\section{References}

1. Axel L, Dougherty L (1989) MR imaging of motion with spatial modulation of magnetization. Radiology 171(3):841-845

2. Young AA, Axel L, Dougherty L, Bogen DK, Parenteau CS (1993) Validation of tagging with MR imaging to estimate material deformation. Radiology 188(1):101-108

3. Corin WJ, Swindle MM, Spann JF Jr, Nakano K, Frankis M, Biederman RW, Smith A, Taylor A, Carabello BA (1988) Mechanism of decreased forward stroke volume in children and swine with ventricular septal defect and failure to thrive. J Clin Invest 82(2):544-551

4. Nakano K, Corin WJ, Spann JF Jr, Biederman RW, Denslow S, Carabello BA (1989) Abnormal subendocardial blood flow in pressure overload hypertrophy is associated with pacing-induced subendocardial dysfunction. Circ Res 65(6):1555-1564

5. Biederman RW, Doyle M, Young AA, Devereux RB, Kortright E, Perry G, Bella JN, Oparil S, Calhoun D, Pohost GM, Dell'Italia LJ (2008) Marked regional left ventricular heterogeneity in hypertensive left ventricular hypertrophy patients: a losartan intervention for endpoint reduction in hypertension (LIFE) cardiovascular magnetic resonance and echocardiographic substudy. Hypertension 52(2):279-286

6. Biederman RW, Young AA, Doyle M, Devereux RB, Kortright E, Perry G, Bella JN, Oparil S, Calhoun D, Pohost GM, Dell'Italia LJ (2015) Regional heterogeneity in 3D myocardial shortening in hypertensive left ventricular Hypertrophy: a cardiovascular CMR tagging substudy to the life study. J Biomed Sci Eng 8(3):213-225

7. Young AA, Kramer CM, Ferrari VA, Axel L, Reichek N (1994) Three-dimensional left ventricular deformation in hypertrophic cardiomyopathy. Circulation 90(2):854-867

8. Biederman RW, Doyle M, Yamrozik J, Williams RB, Rathi VK, Vido D, Caruppannan K, Osman N, Bress V, Rayarao G, Biederman CM, Mankad S, Magovern JA, Reichek N (2005) Physiologic compensation is supranormal in compensated aortic stenosis: does it return to normal after aortic valve replacement or is it blunted by coexistent coronary artery disease? An intramyocardial magnetic resonance imaging study. Circulation 112(9 Suppl):I429-36 
9. Power JA, Thompson DV, Rayarao G, Doyle M, Biederman RW (2016) Cardiac magnetic resonance radiofrequency tissue tagging for diagnosis of constrictive pericarditis: a proof of concept study. J Thorac Cardiovasc Surg 151(5):1348-1355

10. Geskin G, Kramer CM, Rogers WJ, Theobald TM, Pakstis D, Hu YL, Reichek N (1998) Quantitative assessment of myocardial viability after infarction by dobutamine magnetic resonance tagging. Circulation 98(3):217-23

11. Reichek N (1999) MRI myocardial tagging. J Magn Reson Imaging 10(5):609-616

12. Everaars $\mathrm{H}$ et al (2021) Functional recovery after percutaneous revascularization of coronary chronic total occlusions: insights from cardiac magnetic resonance tissue tracking. Int J Cardiovasc Imaging. https://doi.org/10.1007/s10554-021-02355-4

13. Overgaard CB, Džavík V, Buller CE, Liu L, Banasiak W, Devlin G, Maggioni AP, Leor J, Burton JR, Reis G, Ruzyllo W, Forman SA, Lamas GA, Hochman JS, Investigators OAT (2013) Percutaneous revascularization and long term clinical outcomes of diabetic patients randomized in the occluded artery trial (OAT). Int J Cardiol 168(3):2416-22

14. Epstein SE, Pichard AD, Kent KM, Satler L, Suddath WO, Lindsay J, Waksman R (2007) The late open-coronary artery hypothesis: dead, or not definitively tested? Am J Cardiol 100(12):1810-4

15. Marber MS, Brown DL, Kloner RA (1996) The open artery hypothesis: to open, or not to open, that is the question. Eur Heart J 17(4):505-509

16. Wang N, Fulcher J, Abeysuriya N, Adams M, Lal S (2018) Predictors of successful chronic total occlusion percutaneous coronary interventions: a systematic review and meta-analysis. Heart 104(6):517-524

Publisher's Note Springer Nature remains neutral with regard to jurisdictional claims in published maps and institutional affiliations. 